\title{
Building Graphical Model Based System in Sensor Networks
}

\author{
Dongyu Shi, Jinyuan You, and Zhengwei Qi \\ Department of Computer Science and Engineering Shanghai Jiao Tong, \\ University, Shanghai 200030, P.R. China \\ sshidy@yahoo.com.cn, \{you-jy, qi-zw\}@cs.sjtu.edu.cn
}

\begin{abstract}
Consisting of a large number of sensing and computational devices distributed in an environment, a sensor network can gather and process data about a physical area in real time. Due to the limited computing power in each sensor, limited bandwidth connections, limited storage and other limitations, how to deal with the data and uncertainty knowledge is one of the most important and central problems in such kind of distributed systems. This paper presents a graphical model based intelligent system that can model the uncertainty knowledge in sensor networks. This system uses belief messages as a basis for communication. We focus on parameter learning process for building the model, and experiments are presented.
\end{abstract}

\section{Introduction}

Advances in computing and communication over wired and wireless networks have resulted in many pervasive distributed computing environments, such as Internet, local area networks, ad hoc wireless networks, and sensor networks. These environments often come with different distributed sources of data and computation. Sensor networks are a new kind of distributed system, which are fast developing in recent years. They can sense certain phenomena in an environment, while gathering data in real time for further analysis. They consist of a large number of low-cost, lowpower multifunctional computational devices that can be easily deployed in the environment.

Sensor networks are typically used in one of the two modes of operation: either the data from the sensors are extracted from the network and shipped to a server for offline processing; or the information obtained from the sensors is aggregated using local operations in real time within the network itself. How to deal with the "raw" data is one of the central questions in sensor network. Since the bandwidth connections are limited, the extraction of complete data sets can be very expensive, requiring large amounts of communication that drains the energy of these devices. So it is very attractive for mining knowledge from "raw" data locally to reduce communication. But when the data processed online within the network, what form should the information take? How we can compute the uncertainty knowledge from the data? And how should we organize the overall flow of information in a distributed fashion? 
In this paper we use a probabilistic graphical model based intelligent system to solve these problems. Graphical models have become increasingly popular as means to structure uncertainty knowledge in complex domains and thus to facilitate reasoning in such domains. Bayesian Networks and Markov networks are the two most popular kinds of graphical models that have been widely used in uncertainty problems. In many applications, the main task is to form beliefs about the state of the distributed network system based on the collected sensor data. There are some works $[5,7]$ that have been done in this area, and their model has been proved very effective in uncertainty knowledge representation. Here we focus on the parameter learning process for building the distributed intelligent system.

The rest of the paper is organized as follows: we begin in Section 2 with some background and related work. In Section 3, we describe in detail our graphical model based system in a sensor network. In Section 4, we provide the parameter learning process in the system. Section 5 shows the experimental results and the conclusion.

\section{Background and Related Work}

In this section we first give a brief review of probabilistic graphical models, and then introduce the sensor networks and related works about modeling and mining data in sensor networks and other distributed systems.

\subsection{Graphical Models}

A graphical model is a family of probability distributions defined in terms of a directed or undirected graph. The nodes in the graph are identified with random variables, and the (lack of) arcs represent conditional independence assumptions. Hence they provide a compact representation of joint probability distributions [4].

Undirected graphical models are also called Markov networks [8]. They have a simple definition of independence: two (sets of) nodes A and B are conditionally independent given a third set $\mathrm{C}$, if all paths between the nodes in $\mathrm{A}$ and $\mathrm{B}$ are separated by a node in C. By contrast, directed graphical models are also called Bayesian Networks (BNs). They are directed acyclic graphs. They have a more complicated notion of independence, which takes into account the directionality of the arcs. Bayesian network shows the causal relations among its variables. Once completed, both Bayesian network and Markov network can be used to derive the posterior probability distribution of one or more variables using an inference process, with the observed particular values for other variables in the network, or to update previous conclusions when new evidence reaches the system.

\subsection{Sensor Networks and Distributed Data Processing}

The widespread distribution and availability of small-scale sensors, actuators, and embedded processors is transforming the physical world into a computing platform. Sensor networks that combine physical sensing capabilities such as temperature, light, or seismic sensors with networking and computation capabilities will soon become ubiquitous. Applications range from environmental control, warehouse inventory, and health care to scientific and military scenarios. 
Sensor networks are much more tightly integrated with the environment than previous distributed systems. Instead of relying on a small number of interfaces, every (sensor) node in the system is embedded with and in contact with the environment. On the other hand, they rely fundamentally on computation being done by a large number of the distributed nodes. The sensor nodes are generally low-cost, low-power multi-functional devices that can be easily deployed in the environment. Algorithms for these nodes must be implemented very cheaply. Since communication in today's networks is orders of magnitude more expensive than local computation, it is necessary to use in-network storage and processing to vastly reduce resource usage and extend the lifetime of a sensor network.

Sensor networks naturally have much uncertainty knowledge in their systems. The application of distributed data mining and computation to the system is very important, and becomes a core task. Some works have been done in this area. In [6], Carlos Guestrin etc. present distributed regression as an efficient and general framework for in-network modeling of sensor data. In [5], C. Crick and A. Pfeffer use Loopy Belief Propagation as a basis for communication in sensor networks. In [7], M.A. Paskin and Guestrin discussed robustness of probabilistic inference in distributed systems, especially in sensor networks. There are also some works about using probabilistic networks in distributed data mining. In [10], [3], K. Sivakumar and R. Chen discussed Bayesian Network structure learning from distributed data. And in [2], they provide a new algorithm for learning parameters of a $\mathrm{BN}$ in a distributed way.

\section{Modeling Data in a Sensor Network as a Graphical Model}

There is a great deal of uncertainty in sensor network systems. Signals detected at physical sensors have inherent uncertainty, and they may contain noise from the environment. Sensor malfunction might generate inaccurate data, and unfortunate sensor placement (such as a temperature sensor directly next to the air conditioner) might bias individual readings. Reasoning under uncertainty to form coherent beliefs is a major task in sensor network systems.

Here we build a graphical model to deal with the task in a simulated "FireDetection" sensor network. It is supposed to detect fire from sensors collecting temperature and light in a wide area. Sensor networks and sensor nodes have many limitations [1], and one of the general proposed design is the Hourglass architecture [9]. It envisions 4 kinds of nodes: sensor nodes, data nodes, communication nodes and processing nodes. In our model we consider three kinds of nodes:

- Sensor nodes are deployed in the whole target area and responsible for collecting and storing raw sensed data. Each sensor individually provides a reading for a state variable at a particular point. The sensor properties may also affect the reading. Sensor nodes only communicate with the local processing node.

- Processing nodes perform some computation on the data within a local network. Here we divide the whole sensor area into a series of sub-area. In each sub-area there's a local network with a processing node examining a set of sensor nodes. It should form beliefs about high-level variables (such as fire, temperature and light in the sub-area) from sensor readings. The communication between sub-areas will be 
performed by the neighbor processing nodes. And finally, the network of the processing nodes will send information to the central management node.

- The central management node deals with central tasks.

Fig.1 shows an example of a sub-area network. The Fire, Temp and Light are highlevel variables that need to be compute in a processing node. The others are local variables. The "SS1" means variables related to local SenSor node 1, similar with "SSn". We model the sub sensor network as a Bayesian network. The directed edges show the dependent relationships between variables. The high-level variables in adjacent areas are highly correlated. We use a Markov network to represent the relationships between high-level variables in adjacent processing nodes.

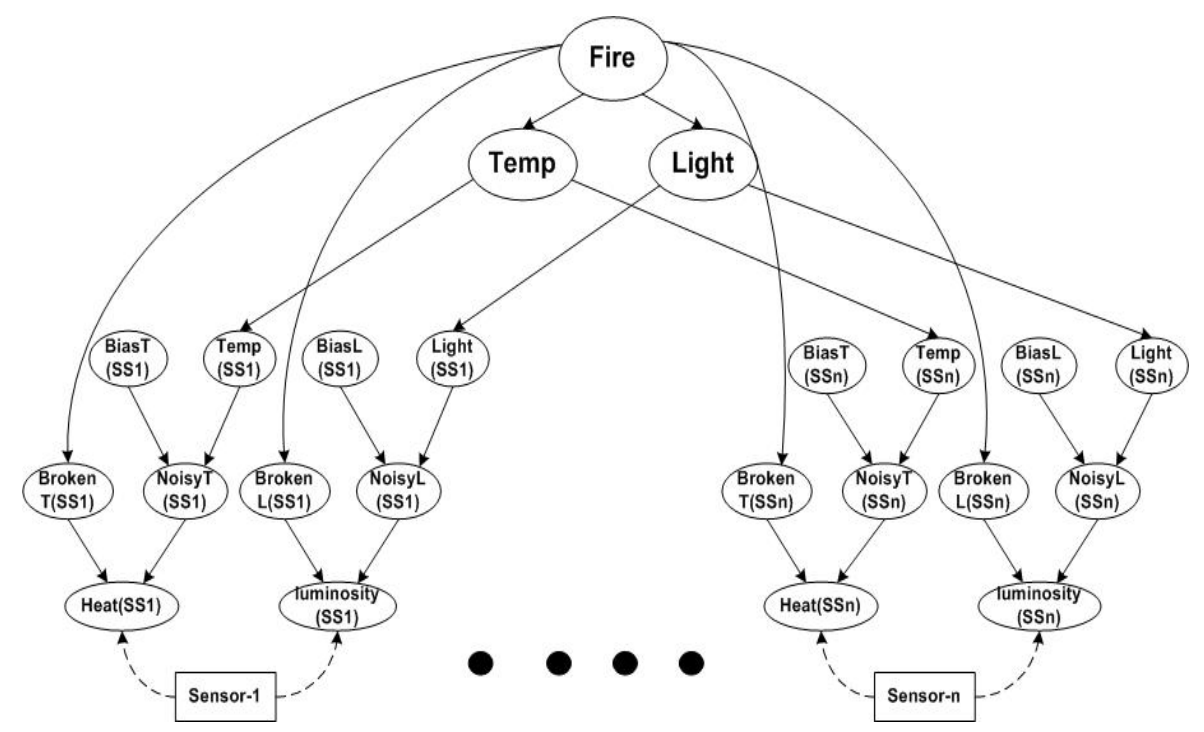

Fig. 1. A local Bayesian network with a processing node and $\mathrm{n}$ sensor nodes

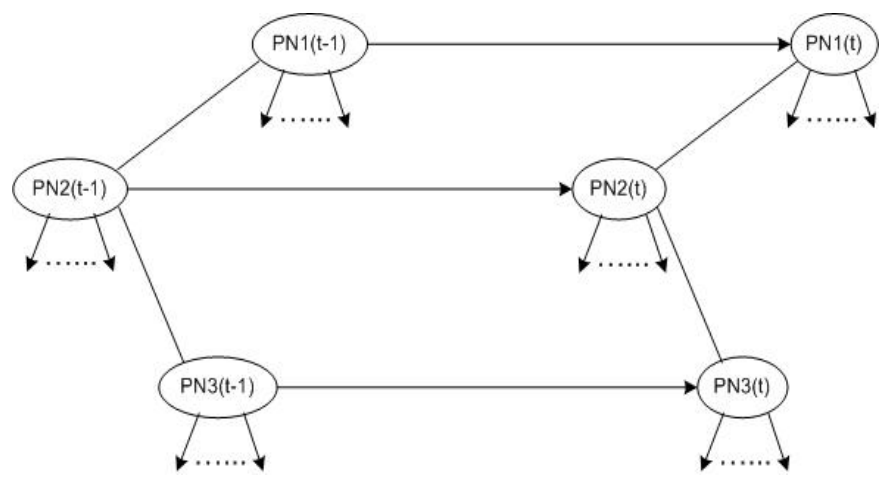

Fig. 2. The dynamic model of the state change between time $t-1$ and $t$ 
Since the environment is changing by time, the state of a sensor network will change dynamically. Here we propose a dynamic model for the whole system. Fig. 2 shows the dynamic model of the communication between processing nodes. The undirected edges between processing nodes (PN1, PN2...) compose a Markov network. There is a directed edge from each processing node at time point t- 1 to the corresponding processing node at time t. So the whole model reveals the state change of the hybrid graphical model between time $\mathrm{t}-1$ and $\mathrm{t}$.

\section{Parameter Learning Process}

Learning parameters of a probabilistic graphical model in a distributed way is one of the major topics in Distributed Data Mining. How to apply the learning process in a sensor network environment can be a critical technique for solving complicated uncertainty problems. In this model, we must first decide the parameters of the distributed graphical model before we make inference and send results to central nodes. Other than arbitrarily decide the parameters, we generate a set of training data samples, and try to learn them within the network.

Since data is distributed among different sensors, we present a collective strategy to learn the parameters in the hybrid model with local Bayesian networks and highlevel Markov networks. The primary steps are in the following:

\subsection{Learning Parameters of Local BN}

At each processing node in a sub-area, learn the variables involved in local BN model based on local data set. We can use the Maximum Likelihood (ML) method to learn the network parameters. And the likelihood function is as follows:

$$
\begin{aligned}
L(\theta: D) & \left.=\prod_{m} P\left(x_{1}[m], \ldots, x_{n}[m]\right): \theta\right) \\
= & \prod_{i} \prod_{m} P\left(x_{i}[m] / P a_{i}[m]: \theta_{i}\right) .
\end{aligned}
$$

$m=1 . . . M$ is the number of sample data sets. Taking Fig. 1 as an example, a complete sample data set includes Heat $(\mathrm{SS} i)$ and luminosity $(\mathrm{SS} i)$ from readings of sensor node $i, \operatorname{Temp}(\mathrm{SS} i)$ and $\operatorname{Light}(\mathrm{SS} i)$ from measuring the state of sensor node $i$, assessment of $\operatorname{Bias}(\mathrm{SS} i)$, setting of BrokenT(SSi) and BrokenL(SSi), all with $i$ from 1 to n; and Temp, Light, Fire from measuring the state of the sub-area. We put all the sample data sets into Equation. 2 according to the network structure of parent-child relationships $(P($ child/Parent $))$, and learning the parameters of the local BN offline.

\subsection{Select Samples for Parameter Learning of High-Level Markov Network}

At each processing node PNk, based on the local $\mathrm{BN}$, we can compute the joint distribution of the high-level variables. Based on this joint distribution, a subset of samples is selected. Let Icol $_{k}$ denote the set of indices of these samples. Then we use the samples to learn parameters of the Markov network among high-level variables of each local BN. 
Here we first discuss the sample selection process. Let $l_{A}($.$) and l_{B}($.$) denote the$ estimated likelihood function involving the local variables at neighbor sub-area $A$ and $B$ respectively. The observations at each local network are ranked based on how well it fits the local model using the local likelihood functions. The observations at $A$ with large likelihood under $l_{A}($.$) are evidence of "local relationships" between variables in$ $A$, whereas those with low likelihood are possible evidence of "cross relationships" between variables across sub-networks. Let $H L$ and $L L$ denote high-level variable sets and sets of other variables in a local network respectively. Consider the marginal distribution of $P(H L)=\sum_{L L} P(H L, L L)$. Therefore, if a sample configuration for $H L$ variables has low likelihood value under $P(H L)$, then there is at least one configuration of $L L$ variables such that the corresponding joint configuration $(H L, L L)$ has low likelihood value under $P(H L, L L)$.

We set a threshold $\boldsymbol{T}$ under $P(H L)$ for each local network and choose the samples whose likelihood is lower than this threshold. As discussed above, these samples are the main part of samples whose likelihood is small under $P(H L, L L)$. Then we introduce a random sampling for the samples whose likelihood is higher than the threshold. We put the two parts of selected sample data sets together, and put them into the high-level Markov network parameter learning. The reason why we do not choose samples under $P(H L, L L)$ is that the total number of configurations $(H L, L L)$ is prohibitively large, but a node in a sensor network has limited memory and computing power.

\subsection{Learning Parameters of High-Level Markov Network}

As the whole network structure is known, and the samples in every local network are chosen, theoretically we can transmit all the samples to central management node and obtain the values of each variable which maximizes the likelihood of the samples. But due to the limited bandwidth connection of sensor network, it will not be feasible. Besides, if the number of sensors is very large, the computation can be a heavy burden for the central node. So we consider learning the parameters within the network. Since we model the network among processing nodes a Markov network, if it has no cliques (fully connected graphs) containing more than 2 nodes, the joint probability distribution over the states of all processing nodes can be decomposed into the product of pair-wise interactions between adjacent nodes. And then we can just exchange local samples between adjacent processing nodes, and use Maximum Likelihood method to learn the parameters locally by processing nodes. Assuming that the number of total selected sets is Selec, the likelihood function of the whole Markov network is as follows:

$$
\begin{array}{r}
\left.L(\theta: D)=\frac{1}{Z} \prod_{m=1}^{\text {Selec }} \psi\left(H L_{1}[m], \ldots, H L_{n}[m]\right): \theta\right) \\
=\frac{1}{Z} \prod_{j} \prod_{m=1}^{\text {Selec }} \psi_{j}\left(H L_{j 1}[m], H L_{j 2}[m]: \theta_{j}\right) .
\end{array}
$$

$m=1$...Selec is the number of sample data sets; $j 1$ and $j 2$ are adjacent nodes that are connected by edge $j ; Z$ is a normalization constant. Taking Fig. 2 as an example, the 
clique functions $\prod_{m=1}^{\text {Selec }} \psi_{1}\left(H L_{1}[m], H L_{2}[m]\right)$ and $\prod_{m=1}^{\text {Selec }} \psi_{2}\left(H L_{2}[m], H L_{3}[m]\right) \quad$ will be included in Equation.4.

If we carefully design the network structure, we can apply this method to the system. In fact in a sensor network, any 3 processing nodes being fully connected may not be necessary. Even when there are cliques containing 3 or more nodes, we can use the above decomposition method as an approximation, and it tend to be a good approximation.

Combining the local BN models and the high-level Markov network with all the parameters, the whole hybrid graphical model can be built. After all these work, we already obtain all the parameters of the network structure. But this is only a snapshot model at a certain time. To obtain a dynamic model we should compute high-level parameter changing in processing nodes between two time slices, and that is the probability $P\left(H L_{i}^{(t)} / H L_{i}^{(t-1)}\right)$. Here we take the assumption that it is a Markov process, the state at $t$ is only affected by the state at $t-1$. We continuously observe the state changing in different periods, and collect data to obtain the probability distribution.

Until now the whole intelligent system for the sensor network has been built. We can apply probabilistic inference techniques to this system, sending beliefs rather than raw data in the sensor network, and get "smart" results of the state in the area that the sensors deployed. We should notice that the probabilistic inference problem in a sensor network environment has already been discussed in [5] by C. Crick and A. Pfeffer. Although our system is different in some kind (like dynamic property), and the inference process requires some further analysis, the feasibility and efficiency are showed in the experiment.

\section{Experiments and Conclusions}

In our first experiment, we simulate a sensor network of 3 processing nodes and 14 sensor nodes in networks. To test the effectiveness of our parameter learning method, we generate 500 sets of sample data. We first compute the parameters in a central way
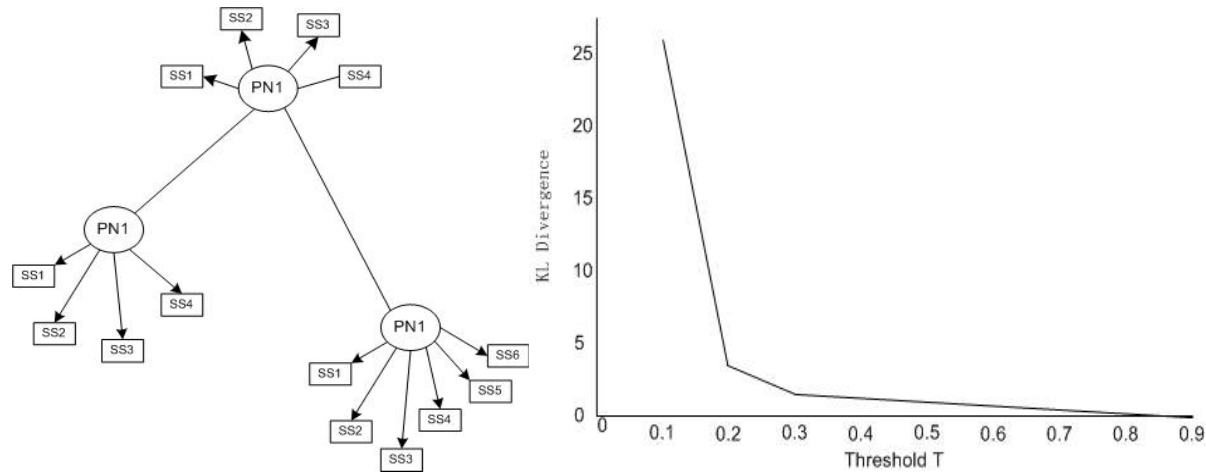

Fig. 3. The network structure and KL-divergence between two joint probability distributions 
using Maximum Likelihood method, and then compare them to the parameters we learned in the distributed way described in section 4. Fig. 3 shows the network structure and the Kullback-Leibler Divergence (or KL distance) between the two joint probability distributions as the threshold $\boldsymbol{T}$ in the second step of the learning process increases.

In another experiment there are 8 local networks, each with a processing node and 3 to 7 sensor nodes, collecting data from more real world environment. The structure of processing nodes is show in Fig.4. The KL divergence between the probability distributions estimated based on our distributed parameter learning method and the parameter obtained using a centralized ML approach is computed. In particular, we illustrate the results for the probability distributions at two different nodes PN3 and PN6. Take PN6 as an example, we compute the sum over all the possible values of its neighbor nodes, of the KL distance of the probability distribution between distributed approach and central approach. The expression is as follows:

$$
\begin{gathered}
\sum_{H L_{5}, H L_{7}, H L_{8}} K L\left(P_{c n t r}\left(H L_{6}\right), P_{d s t r}\left(H L_{6}\right)\right) . \\
P_{c n t r}\left(H L_{6}\right)=\frac{1}{Z_{c n t r}} \psi_{c n t r}\left(h l_{5}, H L_{6}\right) \psi_{c n t r}\left(H L_{6}, h l_{7}\right) \psi_{c n t r}\left(H L_{6}, h l_{8}\right) . \\
P_{d s t r}\left(H L_{6}\right)=\frac{1}{Z_{d s t r}} \psi_{d s t r}\left(h l_{5}, H L_{6}\right) \psi_{d s t r}\left(H L_{6}, h l_{7}\right) \psi_{d s t r}\left(H L_{6}, h l_{8}\right) .
\end{gathered}
$$

$h l_{5}, h l_{7}$ and $h l_{8}$ are a set of certain values that $H L_{5}, H L_{7}$ and $H L_{8}$ take respectively. $\psi_{c n t r}$ and $\psi_{d s t r}$ are clique functions of which the parameters are learned in the central and distributed way respectively.

Fig. 5 depicts the similar KL distance for PN3 and PN6. It shows that generally the KL distance is getting smaller when the threshold getting higher, which means more data are transmitted in the network. But even with a small data communication, it is quite close to that obtained by the centralized approach.

It should be noticed that a real sensor network system could have several hundreds to thousands of sensors. If we learned parameters in a central way, it would be a heavy burden for the server (the central management node), and making it infeasible. So we do not make comparisons between these two ways in a large sensor network system. After learning the temporal parameters - $P\left(H L_{i}^{(t)} / H L_{i}^{(t-1)}\right)$, the whole graphical model based system is build, and can be used for inference and belief propagation.

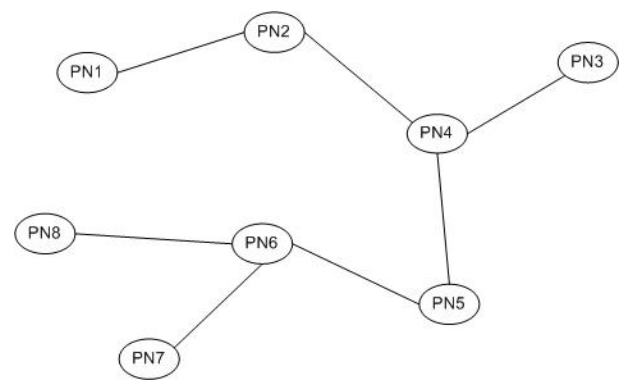

Fig. 4. The structure of processing nodes 

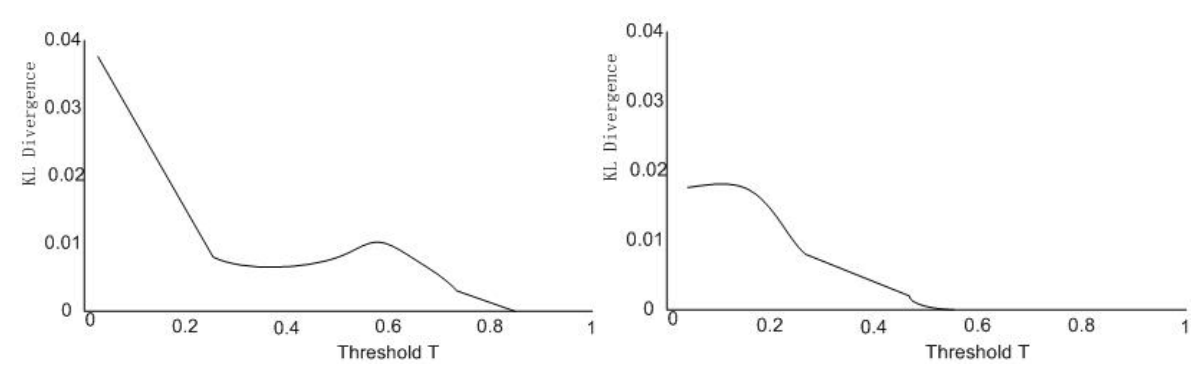

Fig. 5. KL distance between probability distribution learned by our method and central approach of processing node PN3 and PN6

A few tests are run in the system. Comparing to the system in which all the data from the sensors are extracted from the network and shipped to a server for offline processing, our system mainly has advantages in the following two aspects: first, it shows a much quicker response. When there is a simulated fire, it averagely spends $1 / 3$ to $1 / 2$ of time for the GM-based system reporting a fire with the possibility more than $50 \%$. This is because that the GM-based system's response has combined several sensors' readings other than one sensor's. Second, the communication is greatly reduced. We observe that the network delay is much less than raw-data-gathering system. Since the bandwidth is limited, and different local networks have different bandwidth, this quality can greatly improves the performance.

As a conclusion we present an intelligent system that can model the uncertainty knowledge by a dynamic graphical model in sensor networks in this paper. The system uses belief messages as a basis for communication. The parameter learning process for building the model is provided in detail. Experiments have shown that the distributed learning techniques are efficient, and the whole system is feasible and effective. In the future we will apply the system to more large sensor networks.

\section{Acknowledgements}

This paper is supported by the Shanghai Science and Technology Development Foundation under Grant No. 03DZ15027 and the National Natural Science Foundation of China under Grant No. 60173033.

\section{References}

1. Akyildiz, I. F., Weilian Su, Sankarasubramaniam, Y., Cayirci, E.: A survey on sensor networks. Communications Magazine, IEEE Vol. 40, Issue 8, Aug. 2002Page(s):102-114

2. Chen, R., Sivakumar, K.: A new algorithm for learning parameters of a Bayesian network from distributed data. In Proceedings of the 2002 IEEE International Conference on Data Mining, pages 585-588, 2002

3. Chen, R., Sivakumar, K., Kargupta, H.: Collective Mining of Bayesian Networks from Distributed Heterogeneous Data. Knowl. Inf. Syst. 6(2): 164-187 (2004) 
4. Cowell, R., Dawid, P., Lauritzen, S., Spiegel, H.D.: Probabilistic Networks and Expert Systems. Springer, 1999

5. Crick, C., Pfeffer, A.: Loopy belief propagation as a basis for communication in sensor networks. In Proc. of the 19th Conference on Uncertainty in AI (UAI-2003), 2003

6. Guestrin, C., Thibaux, R., Bodik, P., Paskin, M., Madden, S.: Distributed regression: an efficient framework for modeling sensor network data. In Proc. of Information Processing in Sensor Networks 2004 (IPSN-04), 2004

7. Guestrin, C., Paskin, M., Madden, S.: Robust Probabilistic Inference in Distributed Systems. In Proc. of the 20th Conference on Uncertainty in AI (UAI-2004), 2004

8. Pearl, J.: Probabilistic Reasoning in Intelligent Systems: Networks of Plausible Inference. Morgan and Kaufmann, San Mateo, CA, (1988)

9. Shneidman, J., Choi, B., Seltzer, M.: Collecting data for one hundred years. Technical report, Division of Engineering and Applied Science, Harvard University, 2002

10. Sivakumar, K., Chen, R., Kargupta, H.: Learning Bayesian Network Structure from Distributed Data. In Proceedings of the 3rd SIAM International Data Mining Conference, pages 284-288, San Franciso, CA, May 2003 